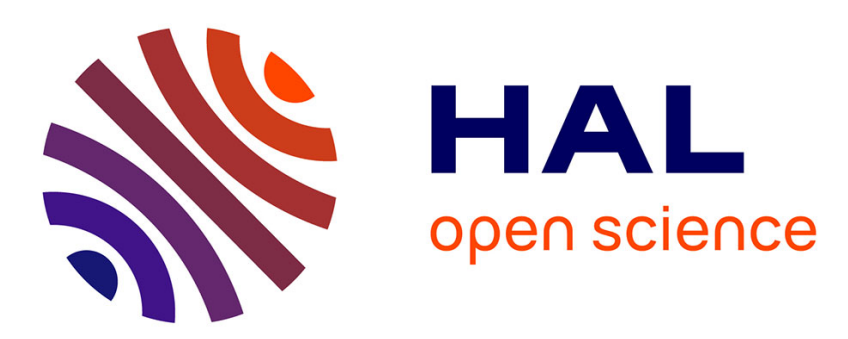

\title{
Analytical solutions of the heat diffusion equation for 3 omega method geometry
}

Jean Yves Duquesne, D. Fournier, Christian Frétigny

\section{To cite this version:}

Jean Yves Duquesne, D. Fournier, Christian Frétigny. Analytical solutions of the heat diffusion equation for 3 omega method geometry. Journal of Applied Physics, 2010, 108 (8), pp.086104. 10.1063/1.3486441 . hal-00544156

\section{HAL Id: hal-00544156 \\ https://hal.science/hal-00544156}

Submitted on 7 Dec 2010

HAL is a multi-disciplinary open access archive for the deposit and dissemination of scientific research documents, whether they are published or not. The documents may come from teaching and research institutions in France or abroad, or from public or private research centers.
L'archive ouverte pluridisciplinaire HAL, est destinée au dépôt et à la diffusion de documents scientifiques de niveau recherche, publiés ou non, émanant des établissements d'enseignement et de recherche français ou étrangers, des laboratoires publics ou privés. 


\title{
Analytical solutions of the heat diffusion equation for $3 \omega$ method geometry
}

\author{
J.-Y. Duquesne ${ }^{1}$, D. Fournier ${ }^{1}$, and C. Frétigny ${ }^{2}$ \\ ${ }^{1}$ Institut des NanoSciences de Paris, UMR 7588, CNRS, \\ UPMC, 140, rue de Lourmel, F-75015 Paris - France \\ ${ }^{2}$ laboratoire de Physico-Chimie des Polymères et des Milieux Dispersés, \\ UMR 7615, UPMC, CNRS, ESPCI, \\ 10 rue Vauquelin, F-75005 Paris, France
}

\begin{abstract}
" $3 \omega$ " experiments aim at measuring thermal conductivities and diffusivities. Data analysis relies on integral expressions of the temperature. In this paper, we derive new explicit analytical formulations of the solution of the heat diffusion equation, using Bessel, Struve and Meijer-G functions, in the $3 \omega$ geometry for bulk solids. These functions are available in major computational tools. Therefore numerical integrations can be avoided in data analysis. Moreover, these expressions enable rigorous derivations of the asymptotic behaviors. We also underline that the diffusivity can be extracted from the phase data without any calibration while the conductivity measurement requires a careful one.
\end{abstract}


The so-called $3 \omega$ method is a well-known method for the measurement of thermal conductivity in bulk and thin film solids [1] [2] [3] [4] [5]. A finite-width metallic strip is designed on the surface of the sample and acts both as a heater and a temperature sensor. It is fed with an AC-current. The heating power has an AC component which generates a thermal pseudo-wave in the sample, at double frequency. The temperature of the strip and, consequently, its resistance are then modulated at double frequency. An harmonic voltage at triple frequency results in and is used to measure both amplitude and phase of the pseudothermal wave, at the surface. For that purpose, a careful calibration of the setup is required. From these values, recorded as a function of frequency, both thermal conductivity and diffusivity can in principle be derived. The theoretical basis for the analysis of the data relies on an integral expression of the temperature. Approximate expressions have been derived in the low frequency [2] and high frequency regimes [6]. Series expansions and semianalytical solutions of the temperature have been put forward recently in the case of a finite thickness strip on a finite solid [7] [8]. In the present paper, we consider a semi-infinite solid and we derive analytical expressions for the temperature distribution at the surface and for the mean temperature of the metallic strip (neglecting the strip thickness and thermal boundary resistance). This formulation uses analytical functions which are all convenient for computation. This greatly simplifies the fitting of experimental data and extraction of thermal parameters since numerical integration is avoided. Moreover, a rigorous derivation of the asymptotic behaviors can be performed and constants which are usually derived numerically are expressed as a function of fundamental mathematical constants.

The theoretical analysis of the " $3 \omega$ " method stems from the infinitely narrow line source of heat, located on the planar surface of a semi-infinite medium $[2,9]$. In that case, the temperature reads:

$$
T_{0}(r)=\frac{P}{\pi \Lambda} K_{0}(q r)
$$

where $q=\sqrt{i \omega / D} . \Lambda$ and $D$ are the thermal conductivity and diffusivity of the solid, respectively. $P$ is the magnitude of the power supplied per unit length, at angular frequency $\omega$ (twice the current angular frequency). $r$ is the distance from the line. $K_{0}$ is the zerothorder modified Bessel functions of second kind.

$T_{0}(r)$ can be considered as a Green function for the problems of surface heating invariant along one axis of the surface. For the $3 \omega$ method, let us consider such a source, homogeneous in an infinite strip parallel to the $y$-axis and located at the surface $(-b<x<b)$ (see figure 


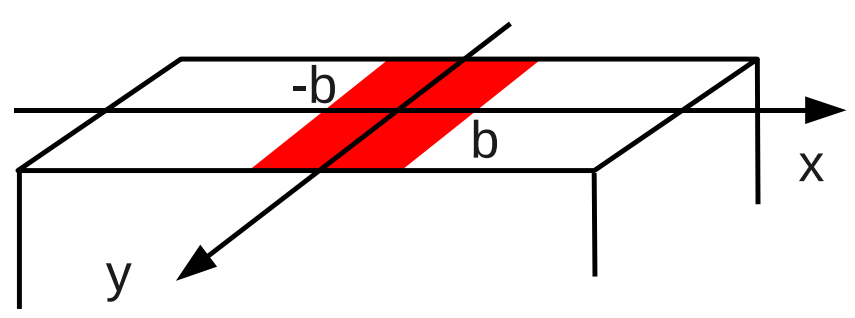

FIG. 1: (Color online) Heat source geometry (red strip) and coordinate axis.

1). The temperature on the surface reads:

$$
T(x)=\frac{P}{2 b \pi \Lambda} \int_{-b}^{+b} K_{0}(q|x-\xi|) d \xi
$$

This expression is usually written as a convolution product and is calculated thanks to Fourier transforms [2]. This leads to an integral expression of the temperature at the surface:

$$
T(x)=\frac{P}{2 b \pi \Lambda} \int_{-\infty}^{+\infty} \frac{\sin (b \lambda)}{\lambda \sqrt{\lambda^{2}+i \omega / D}} e^{+i \lambda x} d \lambda
$$

$3 \omega$ experiments are sensitive to the mean temperature $\langle T\rangle$ of the strip. $\langle T\rangle$ can be estimated by averaging the previous expression across the line [2]. We get:

$$
\langle T\rangle=\frac{P}{\pi \Lambda} \int_{0}^{+\infty} \frac{\sin ^{2} y}{y^{2}} \frac{1}{\sqrt{y^{2}+i \Omega}} d y
$$

where $\Omega$ is the reduced circular frequency:

$$
\Omega=\frac{b^{2} \omega}{D}
$$

Indeed, the integral expression (3) can be replaced with an analytical expression. Equation (2) can be written using Bessel and Struve functions, thanks to the following relation derived from recurrence relations on Bessel functions and from an integral tabulated in [10]:

$$
\int K_{0}(z) d z=\frac{\pi z}{2}\left[K_{0}(z) L_{-1}(z)+K_{1}(z) L_{0}(z)\right]
$$

$K_{n}$ is the nth-order modified Bessel functions of second kind. $L_{n}$ is the nth-order modified Struve function. We define the $\Xi$ function as:

$$
\Xi(z)=K_{0}(z) L_{-1}(z)+K_{1}(z) L_{0}(z)
$$

We get:

$$
\begin{aligned}
T(x)= & \frac{P}{4 \Lambda}\left[(1-X) \Xi\left(\sqrt{i \Omega(1-X)^{2}}\right)\right. \\
& \left.+(1+X) \Xi\left(\sqrt{i \Omega(1+X)^{2}}\right)\right]
\end{aligned}
$$




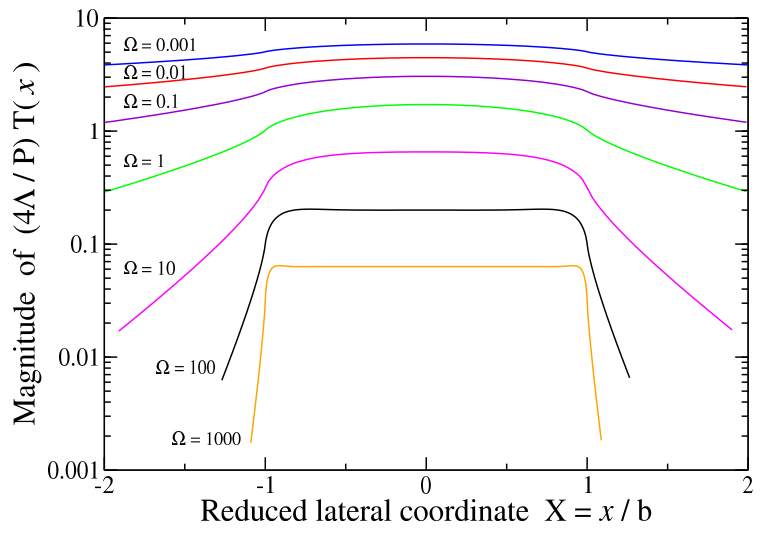

FIG. 2: (Color online) Temperature of the strip: Magnitude of $(4 \Lambda / P) T(x)$ versus the reduced coordinate $x / b$, for different reduced circular frequencies $\Omega$. Equation (8).

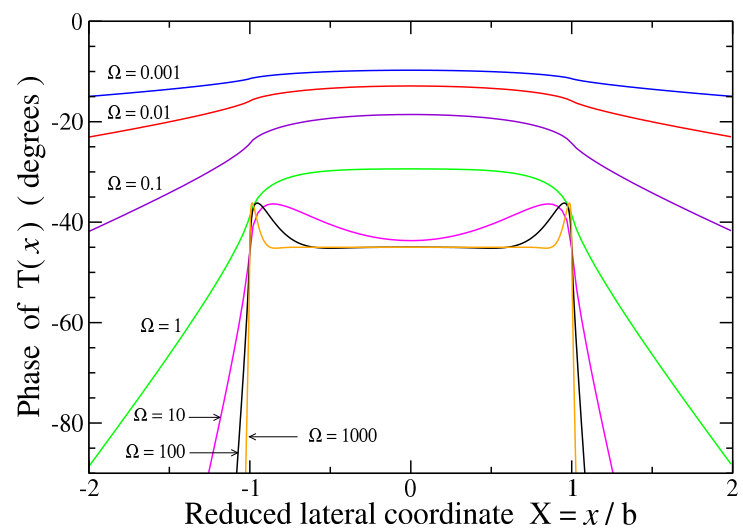

FIG. 3: Temperature of the strip: Phase of $T(x)$ versus the reduced coordinate $x / b$, for different reduced circular frequencies $\Omega$. Equation (8).

where $\mathrm{X}$ is the reduced coordinate.

$$
X=\frac{x}{b}
$$

At the center of the strip $(x=0)$ :

$$
T(0)=\frac{P}{2 \Lambda} \Xi(\sqrt{i \Omega})
$$

Figures 2 and 3 display the magnitude and phase of $T(x)$, computed with equation (8) [11]. Indeed, we have plotted the reduced magnitude $(4 \Lambda / P) T(x)$ and the phase of $T(x)$ versus the reduced coordinate $X$, for different values of the reduced circular frequency $\Omega$.

The integral expression (4) of the mean temperature can also be replaced with an analytical expression. Starting from equation (2), the average temperature of the line can be 


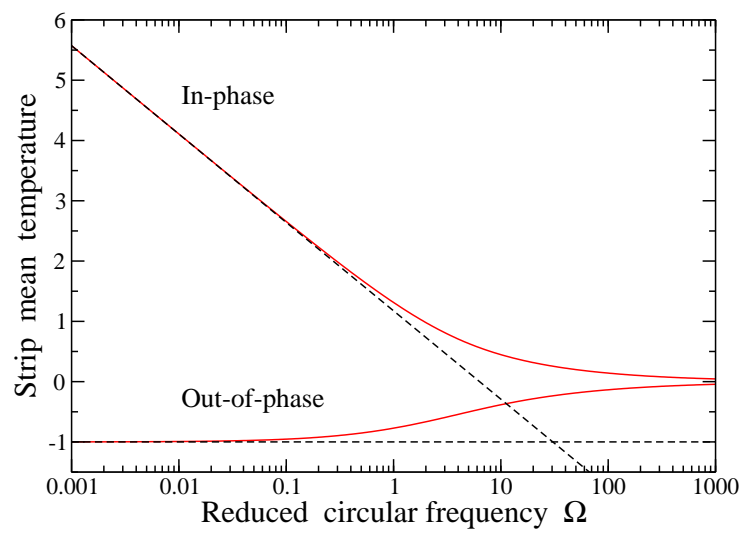

FIG. 4: (Color online) Strip mean temperature versus reduced circular frequency $\Omega$. In-phase and out-of-phase components of $(4 \Lambda / P)<T>$. Solid line: full expression. Equation (14). Dashed line: low frequency approximation. Equation (15).

written as:

$$
\langle T\rangle=\frac{P}{4 b^{2} \pi \Lambda} \int_{0}^{2 b} \int_{0}^{2 b} K_{0}(q|u-v|) d u d v
$$

The integrand is invariant under $u$ and $v$ interchange. By symmetry, the integral is twice the integral evaluated on a rectangular triangle:

$$
\langle T\rangle=\frac{P}{2 b^{2} \pi \Lambda} \int_{0}^{2 b} d u \int_{0}^{u} K_{0}(q(u-v)) d u d v
$$

After variables substitutions $(t=u / 2 b$ and $z=(u-v) / u)$, we get:

$$
\langle T\rangle=\frac{2 P}{\pi \Lambda} \int_{0}^{1} \int_{0}^{1} t K_{0}(r z t) d t d z
$$

where $r=2 q b=2 \sqrt{i \Omega}$. This double integral is related to a Meijer G-function [12]. We get:

$$
\langle T\rangle=-\frac{i P}{4 \pi \Lambda \Omega} G_{24}^{22}\left(i \Omega \mid \begin{array}{l}
1, \frac{3}{2} \\
1,1, \frac{1}{2}, 0
\end{array}\right)
$$

Solid lines in figures 4 and 5 display the in-phase component, out-of-phase component, amplitude and phase of the reduced mean temperature $(4 \Lambda / P)\langle T\rangle$, versus reduced circular frequency, computed with equation (14) [11].

Phase frequency dependance can be of interest since this measurement does not require any absolute calibration of the setup but provides an easy way to measure the sample diffusivity. Indeed, as it is clear from the temperature expressions above, phase of the temperature is a universal function of the only reduced frequency $\Omega$. Therefore, the sample 


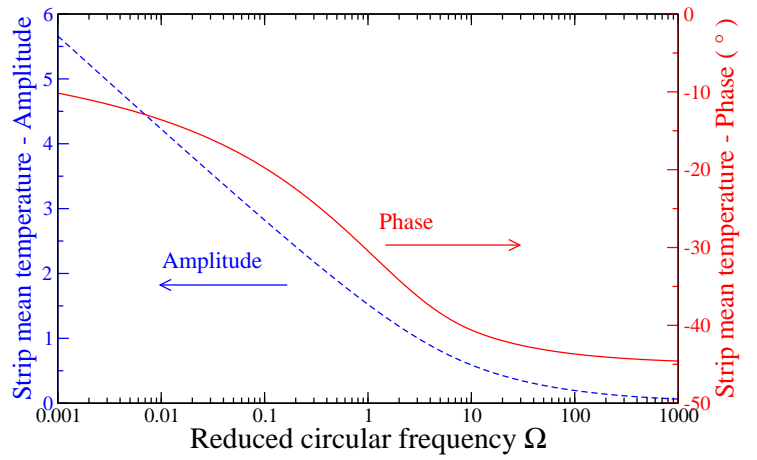

FIG. 5: (Color online) Strip mean temperature versus reduced circular frequency $\Omega$. Solid line: phase of $\langle T\rangle$. Dashed line: amplitude of $(4 \Lambda / P)<T>$. Equation (14).

diffusivity can be extracted with a simple logarithmic shift of the phase curve in figure 5 , along the abscissae, to fit the experimental data.

In both low and high frequency regimes, we can derive useful approximate expressions for $T(x)$ and $\langle T\rangle$, using asymtotic expansions of the the Bessel and Struve functions. Asymptotic analysis of equation (13) gives:

$$
\langle T\rangle \simeq \frac{P}{\pi \Lambda}\left[-\frac{1}{2} \ln \Omega+\frac{3}{2}-\gamma-i \frac{\pi}{4}\right]
$$

for $\omega \rightarrow 0$ (i.e $\omega<<D / b^{2}$ ), and

$$
\langle T\rangle \simeq \frac{P}{2 \Lambda \sqrt{2 \Omega}}(1-i)
$$

for $\omega \rightarrow+\infty$ (i.e. $\left.\omega>>D / b^{2}\right)$.

$\gamma \simeq 0.5772$ is the Euler constant. Equation (15) is well known and is extensively used to measure thermal conductivities. However, we notice the real constant term $(3 / 2-\gamma \simeq$ 0.9228) which is here derived explicitly for the first time. To the best of our knowledge, its approximate value was derived previously numerically.

In the high frequency limit, we may notice that the thermal diffusion length $\sqrt{2 D / \omega}$ becomes much smaller that the heater width. Then, the above result is equivalent to the surface temperature of a uniformly heated semi-infinite medium [9]. However, in this frequency region, thermal behavior can be affected by the thermal load of the heater [7].

We have revisited the theoretical backgrounds of the so-called " $3 \omega$ " method, in the case of a semi-infinite medium with a finite width but vanishing thickness heater. We have derived analytical expressions of the surface temperature and of the mean temperature of the strip 
line. The main benefits are: (1) simplification of experimental data analysis since numerical integration is avoided; the involved Bessel, Struve and Meijer-G functions are implemented in major calculation software and can be easily computed (2) rigorous derivation of the asymptotic regimes at both low and high frequencies.

[1] D. G. Cahill and R. O. Pohl. Phys. Rev. B, 35:4067, 1987.

[2] D. G. Cahill. Rev.of Sci.Instr., 61:802, 1990.

[3] T. Yamane, N. Nagai, S. Katayama, and M. Todoki. J. Appl. Phys., 91:9772, 2002.

[4] C. E. Raudzis, F. Schatz, and D. Wharam. J. Appl. Phys., 93:6050, 2003.

[5] J.-Y. Duquesne. Phys. Rev. B, 79:153304, 2009.

[6] I. K. Moon, Y. H. Jeong, and S. I. Kwun. Rev.of Sci.Instr., 67:29, 1996.

[7] H. Wang and M. Sen. Int. J. of Heat and Mass Transfer, 52:2102, 2009.

[8] S. P. Gurrum, W. P. King, and Y. K. Joshi. J. Appl. Phys., 103:113517, 2008.

[9] H. S. Carslaw and J. C. Jaeger. Conduction of Heat in Solids. Oxford University Press, Oxford, 1959.

[10] M. Abramowitz and I. A. Stegun. Handbook of Mathematical Functions with Formulas, Graphs, and Mathematical Tables, 9th ed. (Dover, New York, 1964)

[11] Computation was performed with mpmath, Python library for arbitrary-precision floatingpoint arithmetic (http://code.google.com/p/mpmath/). Results were also reproduced using commercial computational tools Mathematica (Wolfram Research Inc.) and Maple (Waterloo Maple Inc.).

[12] From the Mellin inversion formula expressing $K_{0}(r z t)$ we have computed directly the double integral and after simplification we obtain an integral form which corresponds to the Meijer definition formula (9.301) of $G_{24}^{22}\left(x \mid \begin{array}{l}1, \frac{3}{2} \\ 1,1, \frac{1}{2}, 0\end{array}\right)$ in [13].

[13] I. S. Gradshteyn and I. M. Ryzhik. in Table of Integrals, Series, and Products, 7th ed., edited by A. Jeffrey and D. Zwillinger (Elsevier, Academic, Amsterdam, Boston, Heidelberg, London, New York, Oxford, Paris, San Diego, San Francisco, Singapore, Sydney, Tokyo, 2007). 